CONSUMO DE SUSTANCIAS ADICTIVAS EN ESTUDIANTES DE PRIMER INGRESO A UN CENTRO UNIVERSITARIO EN JALISCO, MÉXICO.

\author{
CONSUMPTION OF ADDICTIVE SUBSTANCES IN FIRST-INCOME STUDENTS TO A UNIVERSITY CENTER IN \\ JALISCO, MEXICO.
}

Landeros Ramírez Patricia*, Gómez Cruz Zoila*, Núñez-Hernández Alfonsina*, Medina Lerena Miriam Susana*, Jiménez Plascencia Cecilia*.

* Centro Universitario de Ciencias Biológicas y Agropecuarias, Universidad de Guadalajara. México.

Citation: Landeros Ramírez P., Gómez Cruz Z., Núñez-Hernández A., Medina Lerena M.S., Jiménez Plascencia C. (2021) Consumo de sustancias adictivas en estudiantes de primer ingreso a un Centro Universitario en Jalisco, México. Revista Salud Pública y Nutrición, 20 (2), 1-12.

Editor: Esteban G. Ramos Peña, Dr. CS., Universidad Autónoma de Nuevo León, Facultad de Salud Pública y Nutrición, Monterrey Nuevo León, México. Copyright: (C2021 Landeros Ramírez P., et al. This is an open-access article distributed under the terms of Creative Commons Attribution License [CC BY 4.0], which permits unrestricted use, distribution, and reproduction in any medium, provided the original author and source are credited.

Competing interests: The authors have declared that no competing interests exist.

DOI: https://doi.org/10.29105/respyn20.2-1

Recibido: 10 de octubre 2020; Aceptado: 18 de enero 2021

Email: patricia.landeros@academicos.udg.mx 


\title{
CONSUMO DE SUSTANCIAS ADICTIVAS EN ESTUDIANTES DE PRIMER INGRESO A UN CENTRO UNIVERSITARIO EN JALISCO, MÉXICO.
}

\author{
Landeros Ramírez Patricia*, Gómez Cruz Zoila*, Núñez-Hernández Alfonsina*, Medina Lerena Miriam \\ Susana*, Jiménez Plascencia Cecilia*. \\ * Centro Universitario de Ciencias Biológicas y Agropecuarias, Universidad de Guadalajara. México.
}

\section{RESUMEN}

Introducción. el consumo de sustancias adictivas por jóvenes se considera un grave problema de salud pública. Objetivo: conocer la prevalencia del consumo de alcohol, tabaco y otras drogas en estudiantes de primer ingreso a un Centro Universitario en Jalisco, México. Material y Método: se aplicó un cuestionario a los alumnos de nuevo ingreso al Centro Universitario, se obtuvo información sociodemográfica (edad, sexo, promedio de bachillerato, escolaridad de los padres, relación familiar) y consumo de sustancias adictivas (alcohol, tabaco y otras drogas) y se obtuvo su consentimiento informado. Resultados: participaron 608 alumnos, $53.8 \%$ mujeres y $46.2 \%$ hombres, edad promedio $19.1 \pm 1.3$ años, las mujeres tuvieron mejor promedio de bachillerato $(89.45 \pm 5.76)$ respecto a los hombres $(86.12 \pm 6.27)(p<0.001), 49.8 \%$ tuvieron una relación familiar excelente, $59.2 \%$ fumó tabaco alguna vez y $19.8 \%$ fuman en fiestas y reuniones; $92.9 \%$ consumió alcohol en alguna ocasión y $43 \%$ los fines de semana; la droga ilícita más consumida fue marihuana, $24 \%$ ha fumado alguna vez y $1.5 \%$ fines de semana. Conclusiones: En esta investigación, se encontró que un alto porcentaje de jóvenes ha ingerido bebidas alcohólicas, que el fumar está asociado al consumo de alcohol y tener una relación familiar excelente reduce la incidencia del consumo de drogas.

Palabras Clave: Tabaco, alcohol, drogas ilícitas, estudiantes universitarios

\section{ABSTRACT}

Introduction: the consumption of addictive substances by young people is considered a serious public health problem. Objective: to be aware of the consumption of alcohol, tobacco and other drug use in first-time students of the University Center in Jalisco, Mexico. Material and method: a questionnaire was applied to new students at the University Center, and sociodemographic information was obtained (age, sex, high school average, parents' education, family relationship) and consumption of addictive substances (alcohol, tobacco and other drugs) and their informed consent was obtained. Results: 608 students participated, $53.8 \%$ women and $46.2 \%$ men, average age $19.1 \pm 1.3$ years, women had a better high school average ( $89.45 \pm 5.76)$ compared to men $(86.12 \pm 6.27)(p<0.001), 49.8 \%$. They had an excellent family relationship, $59.2 \%$ smoked tobacco at some time and $19.8 \%$ smoke at parties and get together; $92.9 \%$ consumed alcohol at some point and $43 \%$ drank on the weekends; the most widely used illicit drug was marijuana, $24 \%$ had ever smoked and $1.5 \%$ smoked on weekends. Conclusions: In this research, it was found that a high percentage of young people have ingested alcoholic beverages, that smoking tobacco is associated with alcohol consumption and having an excellent family relationship reduces the incidence of drug use.

Key words: Tobacco, alcohol, illicit drugs, college students. 


\section{Introducción}

En el ámbito mundial y nacional existe la preocupación por el incremento constante del consumo de drogas entre jóvenes. La adolescencia en una etapa crítica particularmente vulnerable y los jóvenes pueden desarrollar conductas de riesgo como el consumo de sustancias adictivas (Jiménez, Menéndez e Hidalgo, 2012).

En México, al igual que en muchos otros países, el consumo de sustancias adictivas tanto legales: alcohol y tabaco, como ilegales: marihuana, cocaína, entre otras, constituyen un grave problema de salud pública y es considerado uno de los problemas emergentes que debe ser atendido de manera integral por todos los niveles, en especial por el sector salud y el sector educativo (Zamora-Mendoza, HernándezCastañón, Álvarez-Aguirre, Garza-González y Gallegos-Torres, 2013). La Encuesta Nacional de Consumo de Drogas, Alcohol y Tabaco (ENCODAT) (2017), reporta que el consumo de drogas en la población de 18 a 34 años presentó un incremento significativo durante 2016: cualquier droga pasó de $2.8 \%$ al $5 \%$, drogas ilegales se duplicó (2.3\% a $4.6 \%$ ) y marihuana de $1.9 \%$ a $3.5 \%$; respecto al alcohol, la prevalencia de consumo de alguna vez en la vida en la población adulta es de $77.3 \%$ y en el último año es de $53.3 \%$; en cuanto al tabaquismo, la prevalencia en adultos mexicanos es de $20.1 \%$, encontrándose un mayor consumo de tabaco en hombres $(31.3 \%)$ que en mujeres $(9.8 \%)$.

El uso nocivo del alcohol es uno de los principales factores de riesgo para la salud de la población y en particular en jóvenes ya que disminuye el autocontrol y aumenta los comportamientos de riesgo, su consumo está relacionado con más de 200 enfermedades y trastornos. Se encuentra asociado con la posibilidad de desarrollar problemas de salud como alcoholismo, cirrosis hepática, algunos tipos de cáncer y enfermedades cardiovasculares, así como traumatismos derivados de la violencia y los accidentes de tránsito. Cada año se producen 3 millones de muertes en el mundo debido al consumo nocivo de alcohol, lo que representa un 5,3\% de las defunciones (OMS, 2018).

El tabaquismo es uno de los principales factores de riesgo para el desarrollo de algunas enfermedades crónico-degenerativas, como el cáncer, enfermedades cardiovasculares, pulmonares y efectos perinatales, entre otras. El riesgo de contraer enfermedades causadas por tabaquismo está directamente relacionado con la edad de inicio de su consumo (Plá, Elizarde, Cárdenas, Solares y Nieves, 2016). En México, la edad promedio de inicio de consumo de tabaco es a los 14.3 años (ENCODAT, 2017).

En la mayoría de los países latinoamericanos el consumo de tabaco y bebidas alcohólicas forma parte de las costumbres sociales y culturales. Entre los factores que influyen en el inicio de su consumo se encuentran aspectos de tipo familiar, socioeconómico, cognitivo y emocional. Se ha señalado que el fumar entre jóvenes les da mayor seguridad y confianza para relacionarse e interactuar en grupo, respecto al alcohol, su uso se ve incrementado con la edad, y hay una mayor posibilidad para adquirirlo o consumirlo, ya que entre los jóvenes la percepción del riesgo es muy baja, además de existir una tolerancia para el consumo de alcohol riesgoso, sobre todo cuando el abuso está ligado a la diversión (Telumbre y Sánchez, 2015; Jiménez-Muro, Beamonte, Marqueta, Gargallo y Nerín, 2009).

El ambiente familiar es de suma importancia para el desarrollo de los adolescentes, ya que en esta etapa se presentan los mayores cambios a nivel físico, psicológico y social, que conllevan hacia la vida adulta, se espera que este ambiente sirva para que surjan adultos responsables para interactuar en la sociedad, sin embargo en la familia pueden existir factores que desencadenan diferentes problemas como las adicciones y el consumo de drogas (Herrera-Chávez, Linares-Rubio y Díaz-Barajas, 2018).

El consumo de sustancias psicoactivas constituye una carga sanitaria y social muy significativa, aunque en gran medida prevenible. De acuerdo a la Organización Mundial de la Salud (OMS) más de 450.000 muertes al año se pueden atribuir al consumo de drogas, por lo que señalan, es necesario adoptar medidas para evitar que la mortalidad, morbilidad y discapacidad relacionadas con el uso de drogas y su impacto en el bienestar sigan siendo un problema (OMS, 2016). Los adolescentes se encuentran en una etapa de crecimiento y desarrollo, que los convierte en personas vulnerables para desarrollar patrones de comportamiento que afectan 
su salud y su calidad de vida, incluyendo el consumo $\mathrm{y}$ abuso de sustancias adictivas.

Por otra parte, el ingreso a la universidad es una etapa de la vida, marcada por un aumento del estrés, y es frecuente que no se establezcan conductas saludables (Maturana, 2011; Rodríguez et al., 2016), en estudios previos en este Centro Universitario se ha evidenciado el consumo de sustancias lícitas e ilícitas en estudiantes (Gómez, Landeros, Noa y Patricio y 2017) en este sentido, este trabajo pretende contribuir al conocimiento del riesgo que presenta esta población estudiantil al momento de ingresar a la educación superior y que sirva como herramienta de análisis para generar acciones preventivas, de monitoreo y de promoción a la salud como parte de una educación integral,

Por lo que el presente estudio tuvo como objetivo conocer la prevalencia del consumo de alcohol, tabaco y otras drogas, y analizar la influencia de la relación familiar en dicho consumo, así como conocer el promedio de bachillerato de los estudiantes de primer ingreso a un Centro Universitario en Jalisco, México.

\section{Material y Método}

El presente es un estudio descriptivo y transversal, se aplicaron encuestas a los alumnos de primer ingreso a la universidad, de las licenciaturas de Agronomía, Agronegocios, Biología, Ciencia de los Alimentos y Medicina Veterinaria y Zootecnia del Centro Universitario de Ciencias Biológicas y Agropecuarias (CUCBA) de la Universidad de Guadalajara.

Previa autorización de los respectivos Coordinadores de las Licenciaturas antes mencionadas, se aplicó un cuestionario de manera anónima a todos los alumnos de primer ingreso al Centro Universitario, donde se obtuvo información sociodemográfica (edad, sexo, estado civil, promedio de bachillerato, escolaridad de los padres, responsable del sostenimiento económico), relación familiar (esta se clasificó como excelente, buena, regular y mala, donde cada alumno eligió la respuesta de acuerdo al tipo de relación que tiene con su familia) y consumo de sustancias adictivas (alcohol, tabaco y otras drogas).

Se les explicó el propósito del estudio y se obtuvo su consentimiento informado.
Se excluyeron del estudio: estudiantes que no otorgaron su consentimiento o proporcionaron datos incompletos y menores de 18 años. El protocolo de esta investigación fue autorizado por el Colegio Departamental del Departamento de Salud Pública mediante el Acta núm. SP/159/2019 y la Coordinación de Investigación del Centro Universitario.

Análisis estadístico: Se elaboraron tablas de frecuencia y de contingencia para analizar la distribución de las variables y se evaluó la significancia estadística de las diferencias observadas mediante las pruebas $t$ de Student y Jicuadrada.

\section{Resultados}

El total de la población estudiantil que participó en este estudio fueron 608 alumnos, de los cuales 327 $(53.8 \%)$ fueron mujeres y $281(46.2 \%)$ hombres. Se encontró que los alumnos tenían una edad promedio de $19.1 \pm 1.3$, con mediana de 18 años.

Las licenciaturas con mayor número de alumnos a su ingreso fueron Medicina Veterinaria y Zootecnia $(n=228)$, Biología $(n=174)$ y Agronomía $(n=142)$ seguidas de Ciencia de los Alimentos $(n=36)$ y Agronegocios $(\mathrm{n}=28)$.

La mayoría de los estudiantes refirió ser soltero, vivir y depender económicamente de ambos padres, además de tener una relación familiar excelente.

También, la mayoría de los alumnos mencionó que sus padres cuentan con educación básica ( 9 años de estudio), encontrándose además que $27.0 \%$ de los papás y $22.4 \%$ de las mamás habían concluido una carrera universitaria (ver tabla 1 ). 
Tabla 1. Características sociodemográficas de los estudiantes de primer ingreso al CUCBA

\begin{tabular}{|c|c|c|c|c|c|c|}
\hline & \multicolumn{2}{|c|}{$\begin{array}{l}\text { Mujeres } \\
(\mathrm{n}=327)\end{array}$} & \multicolumn{2}{|c|}{$\begin{array}{l}\text { Hombres } \\
(\mathrm{n}=281)\end{array}$} & \multicolumn{2}{|c|}{$\begin{array}{c}\text { Total } \\
(n=608)\end{array}$} \\
\hline & $\mathrm{n}$ & $\%$ & $\mathrm{n}$ & $\%$ & $\mathrm{n}$ & $\%$ \\
\hline \multicolumn{7}{|l|}{ Edad } \\
\hline 18 a 20 años & 289 & 88.4 & 238 & 84.7 & 527 & 86.7 \\
\hline 21 a 23 años & 23 & 7.0 & 27 & 9.6 & 50 & 8.2 \\
\hline$>23$ años & 15 & 4.6 & 16 & 5.7 & 31 & 5.1 \\
\hline \multicolumn{7}{|l|}{ Estado civil } \\
\hline Soltero & 318 & 97.2 & 274 & 97.5 & 592 & 97.3 \\
\hline Casado & 5 & 1.5 & 3 & 1.1 & 8 & 1.3 \\
\hline Otro & 4 & 1.2 & 4 & 1.4 & 8 & 1.3 \\
\hline \multicolumn{7}{|l|}{ Vive con: } \\
\hline Solo o con amigos & 23 & 7.1 & 20 & 7.1 & 43 & 7.1 \\
\hline Ambos padres & 192 & 58.7 & 194 & 69.1 & 386 & 63.7 \\
\hline Papá o mamá & 87 & 26.6 & 50 & 17.8 & 137 & 22.5 \\
\hline Hermanos & 10 & 3.1 & 9 & 3.2 & 19 & 3.1 \\
\hline Otros & 15 & 4.6 & 8 & 2.8 & 22 & 3.6 \\
\hline \multicolumn{7}{|c|}{ Responsable del sostenimiento económico } \\
\hline Tú mismo & 29 & 8.9 & 41 & 14.6 & 70 & 11.5 \\
\hline Ambos padres & 142 & 43.6 & 136 & 48.4 & 278 & 45.7 \\
\hline Papá o mamá & 152 & 46.6 & 100 & 35.6 & 252 & 41.5 \\
\hline Otros & 4 & 0.9 & 4 & 0.8 & 8 & 1.3 \\
\hline \multicolumn{7}{|l|}{ Relación familiar } \\
\hline Excelente & 167 & 51.0 & 134 & 47.7 & 301 & 49.5 \\
\hline Buena & 132 & 40.4 & 123 & 43.7 & 255 & 41.9 \\
\hline Regular & 24 & 7.3 & 21 & 7.5 & 45 & 7.4 \\
\hline Mala & 2 & 0.6 & 1 & 0.4 & 3 & 0.5 \\
\hline No contestó & 2 & 0.6 & 2 & 0.7 & 4 & 0.7 \\
\hline \multicolumn{7}{|l|}{ Escolaridad materna } \\
\hline Educación básica (9 años) & 127 & 38.8 & 132 & 47.0 & 259 & 42.6 \\
\hline Media superior (3 años) & 93 & 28.4 & 82 & 29.2 & 175 & 28.8 \\
\hline Licenciatura (4 años) & 83 & 25.6 & 53 & 18.8 & 136 & 22.4 \\
\hline Posgrado & 21 & 6.4 & 13 & 4.6 & 34 & 5.6 \\
\hline No contestó & 3 & 0.9 & 1 & 0.4 & 5 & 0.6 \\
\hline \multicolumn{7}{|l|}{ Escolaridad paterna } \\
\hline Educación básica (9 años) & 108 & 33.0 & 115 & 40.9 & 223 & 36.7 \\
\hline Media superior (3 años) & 76 & 23.2 & 68 & 24.2 & 144 & 23.7 \\
\hline Licenciatura (4 años) & 94 & 28.7 & 70 & 24.9 & 164 & 27.0 \\
\hline Posgrado & 30 & 9.2 & 19 & 6.8 & 49 & 8.0 \\
\hline No contestó & 19 & 5.8 & 9 & 3.2 & $r_{28}$ & 4.6 \\
\hline
\end{tabular}

Fuente directa

El promedio de calificaciones obtenido en el bachillerato fue mayor en el grupo de estudiantes que no consumían algún tipo de sustancia adictiva respecto a los consumidores (ver tabla 2 ).

Tabla 2. Comparación del promedio de bachillerato de jóvenes consumidores y no consumidores de sustancias adictivas

\begin{tabular}{|c|c|c|c|c|c|c|}
\hline \multirow[b]{2}{*}{ Sustancia adictiva } & \multicolumn{3}{|c|}{ No consumidores } & \multicolumn{3}{|c|}{ Consumidores } \\
\hline & $\mathrm{n}$ & $\bar{x}$ & DE & $\mathrm{n}$ & $\overline{\mathrm{X}}$ & DE \\
\hline Tabaco* & 250 & 89.4 & \pm 6.08 & 302 & 86.7 & \pm 6.12 \\
\hline Alcohol* & 53 & 90.4 & \pm 5.63 & 502 & 87.6 & \pm 6.22 \\
\hline Marihuana* & 428 & 88.5 & \pm 6.25 & 131 & 86.2 & \pm 5.80 \\
\hline Cocaína* & 528 & 88.1 & \pm 6.12 & 9 & 82.2 & \pm 5.93 \\
\hline Otras drogas & 531 & 88.0 & \pm 6.11 & 13 & 85.1 & \pm 7.60 \\
\hline
\end{tabular}

Fuente directa

$\bar{X}$ : Promedio

DE: Desviación estándar

*T de Student $<0.005$
Consumo de tabaco

En la figura 1 se muestran las características sobre el consumo de tabaco, donde se destaca que los hombres reportaron un consumo significativamente mayor $(\mathrm{p}<0.05)$ que las mujeres respecto al consumo diario y en los últimos 12 meses. En cuanto a la edad de inicio de consumo de tabaco, se observó que las mujeres fumaron por primera vez en promedio a los 16.6 años \pm 1.79 (DE) y los hombres a los $16.4 \pm 2.04$ (DE) años, sin diferencia estadística.

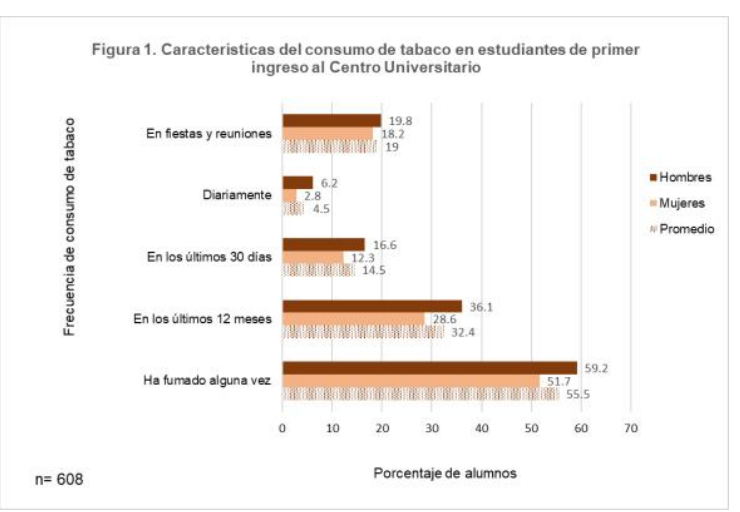

Acerca de la opinión que tienen los jóvenes sobre el consumo de tabaco, éstos mencionaron que fumar es dañino (82\%), es agradable (46\%), está mal visto $(23.6 \%)$, reduce el estrés $(18.2 \%)$, sirve para socializar $(5.0 \%)$, es apetecible $(3.8 \%)$, hace sentir bien $(3.3 \%)$, mejora el estado de ánimo (2.3\%), da seguridad (1.2) y ayuda a estar delgado (1.2\%). Por otro lado, en este estudio $34.3 \%$ de la población estudiantil manifestó que algún miembro de su familia fumaba: papá (15.1\%), mamá (4.8\%), ambos padres $(3.4 \%)$, hermanos $(7.5 \%)$ y otros $(3.5 \%)$.

Además, al analizar las variables: vivir con sus padres y el consumo de tabaco mediante la prueba Jicuadrada, se observó que el vivir con ambos padres reduce el riesgo de fumar, y el vivir únicamente con el padre se asoció con este riesgo con diferencia estadística $(\mathrm{p}=0.021)$.

Consumo de alcohol

Del total de la muestra estudiada, $90.6 \%$ de los alumnos refirió haber consumido alcohol alguna vez en su vida, y $62.9 \%$ ingirió bebidas alcohólicas en los últimos 30 días. Se observó que los fines de semana los hombres presentaron un consumo 
significativamente mayor $(\mathrm{p}<0.001)$ que las mujeres (figura 2). La edad promedio de inicio en el consumo de alcohol en mujeres fue de $16.5 \pm 1.65$ (DE) y en hombres $16.1 \pm 1.87$ (DE). Al preguntarles acerca del número de bebidas alcohólicas que ingieren por ocasión, se encontró que $47.3 \%$ toma 1 a 2 copas, $27.3 \%$ de 3 a $4,13.6 \%$ de 5 a $6,8.4 \%$ de 7 a 9 y $3.5 \%$ ingiere 10 o más copas, siendo el tequila (41.3\%) y la cerveza (37.9\%) las de mayor preferencia.

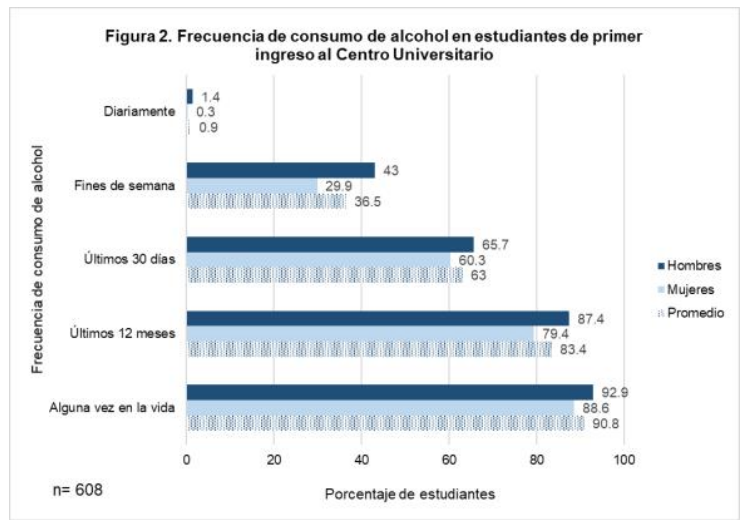

En este estudio, mediante la prueba Ji-cuadrada, se encontró que fumar está asociado al consumo de bebidas alcohólicas $(p<0.001)$ ya que los jóvenes que fuman tienen mayor probabilidad de consumir alcohol.

\section{Consumo de drogas}

La frecuencia de consumo de drogas ilegales (marihuana, cocaína y otras drogas) por los estudiantes, se muestra en la figura 3 , siendo la marihuana la que el mayor porcentaje de estudiantes la consume, observándose en todos los casos una importante disminución cuando se compara la frecuencia de consumo de alguna vez en la vida, año, mes, fines de semana y diariamente.

Entre los principales motivos que llevaron a los jóvenes a consumir algún tipo de droga ilícita se encuentran la diversión (14\%), la curiosidad y la experimentación $(7.5 \%)$ y en menor proporción: aburrimiento $(1.7 \%)$, rebeldía $(1.2 \%)$, aceptación $(0.7 \%)$, problemas $(0.7 \%)$ o porque los demás lo hacen $(0.5 \%)$.

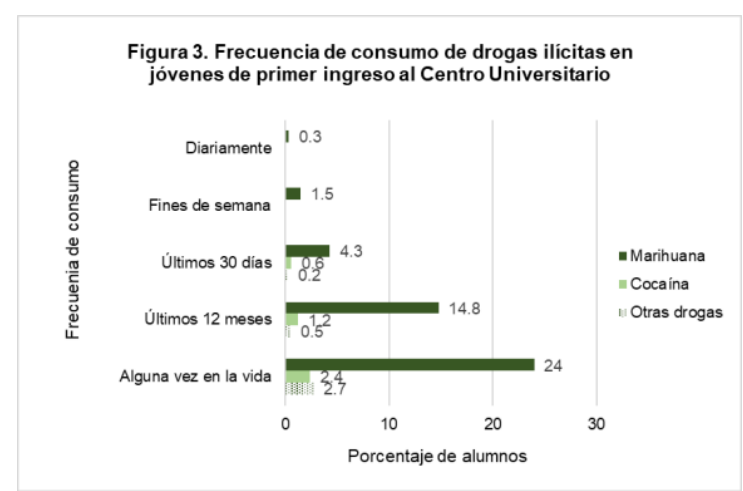

Al analizar mediante la prueba Ji-cuadrada, la relación familiar de los encuestados con el consumo de sustancias adictivas, se observó en todos los casos, que un mayor porcentaje de los jóvenes que no consumen tabaco (54.9\%), alcohol (56.4\%), marihuana $(52.3 \%)$, cocaína $(50.3 \%)$ y otras drogas $(50.3 \%)$ tienen una relación familiar excelente, sin embargo, los que consumen algún tipo de droga mencionó en su mayoría consideran su relación familiar ya sea buena o regular y una pequeña proporción de estos la consideran mala, con diferencia estadística $(\mathrm{p}<0.05)$.

\section{Discusión}

El ingreso a la universidad es una etapa considerada de vulnerabilidad para los jóvenes, debido entre otras cosas a que tienen nuevas experiencias, nuevos lazos de amistad y en algunos casos por la separación de la familia, existen factores socioambientales que pueden influir en el consumo de drogas y en comportamientos de riesgo (Antoniassi y Gaya, 2015). Los adolescentes al estar inmersos en un proceso de maduración y desarrollo son especialmente vulnerables a los efectos de estas sustancias tóxicas (Simón et al., 2019).

En este estudio el consumo de tabaco alguna vez en la vida, es similar al reportado por Telumbre-Terrero, Esparza-Almanza, Alonso-Castillo y AlonsoCastillo (2016) y por González et al. (2019) quienes identificaron una prevalencia de $58.1 \%$. Al analizar el consumo diario de tabaco, se encontró que la prevalencia es menor en mujeres en comparación con los hombres, situación similar a lo reportado por otros estudios (ENCODAT 2017; Chinwong, Mookmanee, Chongpornchai, y Chinwong, 
2018; Reyes-Ríos, Camacho-Rodríguez, FerrelBallestas, Ferrel-Ortega y Bautista-Pérez, 2018) y difiere de Barra et al., (2015), Alvear-Galindo et al., (2015) y Ordás et al., (2017) quienes no encontraron diferencia estadística significativa en el consumo de tabaco según el género. Valdez-Rodríguez, Rodríguez-Olivas, Hernández-Bernadett y TerrazasAltamirano (2019) en un estudio realizado en el Instituto Tecnológico de Chihuahua en México, encontraron que casi la mitad de los estudiantes encuestados son fumadores sociales $(48.48 \%)$, con hábitos de consumo en fiestas y grupos de amigos, en nuestro estudio los datos indican un porcentaje menor (19.0\%). La edad en la que iniciaron a fumar los hombres (16.4 años) y mujeres (16.6 años) concuerda con la edad que reportan (15 a 16 años) Strunin et al., (2017) y Reyes-Ríos et al., (2018), y es superior a la que indican Villatoro et al., (2016), quienes encontraron que los hombres empiezan a fumar a los 12.6 años y las mujeres a los 13.1 años. Existen varios factores que influyen en la edad de inicio en el consumo de tabaco, alcohol y otras drogas, entre ellos factores culturales y contextuales como son la educación de los padres, la externalización de problemas de conducta, disponibilidad del tabaco, el uso de otras sustancias adictivas, situación económica, cuestiones de identidad, etc. (Strunin et al., 2017; Sánchez-Hoil et al., 2017).

Este estudio mostró que la prevalencia en el consumo de tabaco en los últimos 12 meses $(32.1 \%)$ coincide con otras investigaciones (Caravaca et al., 2015 y Rodríguez et al., 2016) y difiere de lo encontrado por Aguilar (2015) y Telumbre-Terrero et al. (2016) quienes encontraron una prevalencia de $21.0 \%$ y $41.6 \%$ respectivamente.

En relación a la opinión sobre el consumo de tabaco, la mayoría de los estudiantes tiene conocimientos acerca de los daños a la salud que causa el fumar, nuestros resultados coinciden con diversos estudios, donde más del $80 \%$ de los jóvenes reconoce que fumar causa problemas de salud (Ortega-Ceballos, Terrazas-Meraz, Arizmendi-Jaime y TapiaDomínguez, 2018; Fernández-Castillo, Molerio, López, Cruz y Grau, 2016; García-García, VázquezGalindo, Hayashida y Dos Santos, 2014), lo cual pudiera ser un factor protector en el consumo de cigarrillos, sin embargo también mencionaron que fumar es agradable, reduce el estrés, sirve para socializar, mejora el estado de ánimo, les proporciona seguridad, y ayuda a estar delgados, estas opiniones coinciden con lo encontrado por Peña y Ávila (2016), Reyes-Ríos et al., (2018), Duarte, Varela, Salazar, Lema y Tamayo (2012) y Valdéz et al. (2019).

Los resultados de este estudio mostraron que el vivir con ambos padres disminuye las probabilidades de fumar de los encuestados, datos semejantes a lo reportado por Maturana, (2011) quien señala que los adolescentes que conviven en familias en las que no hay ningún fumador, tienen más probabilidades de no consumir que aquellos donde uno o varios integrantes si fuman, la relación se intensifica en las familias donde los fumadores son (padre, madre y hermano). Otten, Engels, van de Ven y Bricker, (2007) indican que la estructura familiar influye sobre el consumo de tabaco de los jóvenes, esto puede explicarse debido a que existe una mayor supervisión y responsabilidad, a diferencia de familias con un solo padre.

Respecto al consumo de alcohol alguna vez en la vida y en el último año, nuestros resultados indican una mayor prevalencia $(90.8 \%$ y $83.4 \%$ respectivamente), a lo encontrado en la población mexicana de 18 a 65 años (ENCODAT, 2017), y a lo reportado por Caravaca et al., 2015 (78.4\%) y por Telumbre y Sánchez (2015) (68.9\%), datos similares a los nuestros fueron reportados por Armendáriz et al., (2014) en un estudio realizado en Nuevo León, México, en donde un alto porcentaje de alumnos $(92.2 \%)$ mencionó haber ingerido bebidas alcohólicas en alguna ocasión.

Maturana (2011) y Armendariz et al. (2014) señalan que las reuniones que realizan los jóvenes los fines de semana surgen como una necesidad en el sentido que necesitan reafirmar su identidad y buscan relacionarse consumiendo alcohol y otras drogas para generar vínculos sociales. En nuestra encuesta, la prevalencia de estudiantes que declararon ingerir bebidas alcohólicas los fines de semana fue mayor en hombres (43.3\%) que en mujeres (29.9\%) mostrándose este mismo patrón en otras investigaciones (Poscia et al., 2015; García, Gimenez, Castro, Nebot y Ballester, 2018), por otra parte, existen pocos estudios donde se muestre que las mujeres presentan un mayor consumo (Villareal, Sánchez y Musito, 2013). 
Las evidencias sugieren que el inicio temprano de consumo de bebidas alcohólicas aumenta en gran medida el riesgo de desarrollar trastornos relacionados con el alcohol (Giustino et al., 2018), aunque algunas investigaciones reportan el inicio de consumo a edades tempranas (Villatoro et al., 2016; Navalón y Ruiz, 2017; Castaño-Pérez y CalderónVallejo, 2014), en este estudio la edad de consumo por primera vez fue mayor (16.3 años), lo que es congruente con lo afirmado por González et al, 2019; Telumbre-Terrero et al. 2016). En México, la edad legal para consumir alcohol es a los 18 años, sin embargo, el consumo de esta sustancia está ampliamente disponible y es muy accesible para los jóvenes y existe poca verificación de la edad en establecimientos (Strunin et al., 2017), por otro lado, la ingesta de alcohol es parte de la vida social dado que está presente como vehículo de socialización incluyendo fiestas familiares como son las celebraciones de bautizos, cumpleaños, bodas y festividades con arraigo cultural (Medina-Mora, 2007; Armendariz et al., 2014).

En relación al número de bebidas alcohólicas que ingieren los jóvenes por ocasión, este estudio mostró un consumo menor que lo reportado por TelumbreTerrero et al., (2016) quienes señalan que los alumnos ingieren en promedio hasta 5 copas por ocasión, y coincide con Betancourth-Zambrano, Tacán-Bastidas y Córdoba-Paz (2017), quienes realizaron un estudio en jóvenes universitarios colombianos y encontraron que en promedio ingieren 2 o 3 bebidas embriagantes en un día de consumo de alcohol.

Se encontró que los hombres consumen más cantidad de alcohol que las mujeres lo que concuerda con lo reportado por Ferretti et al., (2018) y por Yadav, Khanuja y Velaga (2020); Caro, García, Acosta, Ibáñez y Delgado, 2015; Barrera et al., 2020) quienes indican que los hombres beben con mayor frecuencia y consumen mayor cantidad de alcohol que las mujeres, esto puede deberse a las diferencias en las características físicas, las mujeres necesitan una cantidad menor de alcohol para alcanzar el mismo nivel de embriaguez que los hombres debido a su menor índice de masa corporal y al metabolismo de alcohol más lento, de acuerdo con Erol y Karpyak, (2015) las mujeres suelen tener menos probabilidades de tener problemas con la bebida.
Los tipos de bebidas alcohólicas más consumidas fueron el tequila y la cerveza, resultado que corresponde con lo descrito en otras investigaciones (Puig-Nolasco, Cortaza-Ramirez y Pillon, 2011; Telumbre y Sánchez, 2014) mientras que otros autores señalan que la cerveza es la preferida por los universitarios (Salcedo, Palacios y Espinosa, 2011; Caro, et al., 2015; Barrera et al., 2020).

En relación al consumo de drogas ilícitas, el tipo de droga más utilizada por los jóvenes encuestados fue la marihuana seguida de la cocaína, resultados que coinciden con Tiburcio et al., (2016), Caravaca, et al., (2015) y Figueredo (2014), al investigar sobre los motivos de su consumo, la mayoría de los alumnos en este estudio indicaron: diversión, curiosidad y experimentación, similar a lo reportado en otras investigaciones (Gámez-Medina, Ahumada-Cortez y Valdez-Montero, 2017; Tirado et al., 2012).

Diversas investigaciones señalan que los entornos familiares desestructurados, con padres que consumen sustancias adictivas son más permisivos, y propician que los hijos adolescentes sean más proclives al consumo de drogas. Simón, et al., (2019) encontraron como factor protector el tener una buena relación con los padres ya que estos se consideran proveedores de afecto y puede contribuir a disminuir el malestar emocional de los jóvenes. En este trabajo se observó que un mayor porcentaje de los estudiantes que no consumen drogas consideran que tienen una relación familiar excelente, lo que concuerda con lo reportado por Caravaca et al., (2015) quienes señalan que jóvenes de familias con estructuras fuertes, con mayor confianza y lazos de comunicación más estrechos, tienen menores probabilidades de consumir drogas que aquellos que viven con falta de comunicación, violencia y desapego. Ruiz-Risueño Ruiz-Juan, y Zamarripa (2012), señalan la importancia de la influencia de los familiares (padres y hermanos concretamente), no solo para el consumo de alcohol sino también el de otras sustancias.

\section{Conclusiones}

En el presente estudio se encontró que un alto porcentaje de jóvenes de primer ingreso al Centro Universitario ha ingerido bebidas alcohólicas, los jóvenes que fuman tienen mayor probabilidad de consumir alcohol, además los estudiantes que 
tuvieron una relación familiar excelente presentaron una menor incidencia en el consumo de sustancias adictivas, siendo esta una etapa importante en la vida de los estudiantes para su futuro personal y profesional, es necesario reflexionar sobre el papel de la Universidad con acciones que permitan prevenir el consumo de drogas, así como la identificación temprana de estudiantes que puedan presentar problemas de adicciones.

\section{Agradecimientos}

Para el desarrollo de este estudio se contó con el apoyo del Programa de Fortalecimiento de Cuerpos Académicos, Convocatoria 2019 de PRODEP, Universidad de Guadalajara.

\section{Bibliografía}

Aguilar, A. (2015). Incidencia de las variables sociodemográficas en los patrones de consumo del tabaco en estudiantes universitarios. Revista Entorno. 59: 69-76. Disponible en https://biblioteca.utec.edu.sv/entorno/index.php/ entorno/article/view/475

Alvear-Galindo, M., Yamamoto-Kimura, L., MoránÁlvarez, I., Rodríguez-Pérez, A., Solís-Torres, C., Varela-Mejía, H., Fajardo-Yamamoto, M. (2015). Tobacco use, alcohol consumption and family history, such as risk of chronic disorders in university students. Rev Med Hosp Gen Méx. 78(2):67-72. DOI: 10.1016/j.hgmx.2015.03.008

Armendáriz, N., Alonso, M., Alonso, B., López, M., Rodríguez, L y Méndez, M. (2014). La familia y el consumo de alcohol en estudiantes universitarios. Ciencia y enfermería. 20 (3): 109118. Disponible https://dx.doi.org/10.4067/S071795532014000300010

Antoniassi, J y Gaya, C. (2015). Implications of the use of alcohol, tobacco and other drugs in the university student's life. Rev Bras Promoc Saúde, Fortaleza. 28 (1): 67-74. Disponible en http://www.bioline.org.br/abstract?bh15018

Barra, L., Fernández, P., Granada, F., Ávila, P., Mallea J., y Rodríguez, Y. (2015). Diagnóstico del consumo de tabaco en estudiantes de pregrado de la Pontificia Universidad Católica de
Valparaíso. Rev Med Chile. 143: 1343-1350. Disponible en: https://scielo.conicyt.cl/pdf/rmc/v143n10/art14.p df

Barrera, C., Vergara, R., Dominguez, D., González, A., Cedeño, D., Solis, D. (2020). Prevalencia y factores asociados al consumo de bebidas alcohólicas en estudiantes del distrito de Las Tablas. Visión Antataura. 4(1): 36-514. Disponible en: http://www.revistas.up.ac.pa/index.php/antataur a/article/view/1395/1152

Betencourth-Zambrano, S., Tacán-Bastidas, L., Córdoba-Paz, E.G. (2017) Consumo de alcohol en estudiantes universitarios colombianos. Rev Univ. $\quad$ Salud.19 (1):37-50. DOI: http://dx.doi.org/10.22267/rus.171901.67. Disponible en: http://www.scielo.org.co/pdf/reus/v19n1/01247107-reus-19-01-00037.pdf

Caravaca, J., Noh. S., Hamilton, H., Brands, B., Gastaldo, D., Miotto, G. (2015) Factores socioculturales y consumo de drogas entre estudiantes universitarios costarricenses. Texto \& Contexto - Enfermagem. 24 (spe): 145-153. Disponible en: http://dx.doi.org/10.1590/010407072015001170014

Caro, Á., García, D., Acosta, R., Ibáñez, E., y Delgado, N. (2015). Consumo de alcohol y factores asociados en estudiantes de instrumentación quirúrgica en una universidad de Bogotá, 2014. Revista Salud Bosque. 5 (2): 25$34 . \quad$ Disponible en: file:///C:/Users/hizoy/Downloads/Consumo_de_ alcohol_y_factores_asociados_en_estudia\%20(2 ).pdf

Castaño-Perez, G y Calderon-Vallejo G. (2014). Problemas asociados al consumo de alcohol en estudiantes universitarios. Rev. Latino-Am. Enfermagem. 22(5):739-46. DOI: 10.1590/01041169.3579.2475. Disponible en: https://www.scielo.br/pdf/rlae/v22n5/es_01041169-rlae-22-05-00739.pdf

Chinwong, D., Mookmanee, N., Chongpornchai, J., \& Chinwong, S. (2018). A Comparison of 
Gender Differences in smoking behaviors, intention to quit, and nicotine dependence among Thai University students. Journal of Addiction. DOI: $\quad$ https://doi.org/10.1155/2018/8081670. Disponible en: https://www.hindawi.com/journals/jad/2018/808 1670/

Duarte, C., Varela, M., Salazar, I., Lema, L., Tamayo, J. (2012). Motivaciones y recursos para el consumo de sustancias psicoactivas en universitarios. Revista Hacia la Promoción de la Salud. 17(1): 92-104. Disponible en http://www.redalyc.org/articulo.oa?id=3091248 94009

ENCODAT. Encuesta Nacional de Consumo de Drogas, Alcohol y Tabaco 2016-2017. (2017). Disponible en https://www.gob.mx/salud\%7Cconadic/acciones -y-programas/encuesta-nacional-de-consumode-drogas-alcohol-y-tabaco-encodat-2016-2017136758

Erol, A., y Karpyak, V. (2015). Sex and genderrelated differences in alcohol use and its consequences: Contemporary knowledge and future research considerations. Drug and Alcohol Dependence. 156, 1-13. Disponible en https://doi.org/10.1016/j.drugalcdep.2015.08.02 3.

Fernández-Castillo, E., Molerio, O., López, L., Cruz, A., y Grau, R. (2016). Percepción de riesgo respecto al consumo de tabaco en jóvenes universitarios cubanos. Revista Cubana de Medicina General Integral. 35(2): 140-152. Disponible en http://scielo.sld.cu/pdf/mgi/v32n2/a01.pdf

Ferretti, F., Pozza, A., Harri, P., Francalanci, C., Gualtieri, G., y Coluccia, A. (2018) Drinking wine to "get high": The influence of awareness of the negative effects among young adults. Adicto. Behav. Rep. 8: 56-61. Disponible en: https://doi.org/10.1016/j.abrep.2018.07.002

Figueredo, P. (2014). Prevalencia del consumo de sustancias psicoactivas en estudiantes universitarios de la Universidad Tecnológica Intercontinental (UTIC) - Región Central. ARANDU UTIC. 1(1):151-178. Disponible en:
file:///C:/Users/hizoy/Downloads/DialnetPrevalenciaDelConsumoDeSustanciasPsicoactiv asEnEst-7332942\%20(1).pdf

Gámez-Medina, M., Ahumada-Cortez, J., y ValdezMontero, C. (2017). Las representaciones sociales del consumo de tabaco, alcohol y otras drogas. Ra Ximhai, 13 (2): 25-37. Disponible en: https://www.redalyc.org/pdf/461/46154510002. pdf

García, M., Giménez, C., Castro, J., Nebot, J., Ballester, R. (2018) ¿Existe relación entre el consumo de alcohol de los padres y el de los adolescentes? Revista Internacional de Psicología del Desarrollo y de la Educación, 2 (1): 229-238. Disponible en: https://www.redalyc.org/articulo.oa?id=3498/34 9856003025

García-García, P., Vázquez-Galindo, L., Hayashida, M., Dos Santos, M. (2014). Prevalence and opinions related to tobacco consumption by university students. Revista Electrónica en Salud Mental, Alcohol y Drogas. 10(3):135-142. Disponible en: http://www.redalyc.org/articulo.oa?id=8033779 2005

Giustino, A., Stefanizzi, P., Ballini, A., Renzetti, D., De Salvia, MA, Finelli, C., Coscia, MF, Tafuri, S., y De Vito, D. (2018). Alcohol use and abuse: A cross-sectional study between Italian adolescentes. J. Prev. Medicina. Hyg. 59: 167$171 . \quad$ Disponible en: https://www.ncbi.nlm.nih.gov/pmc/articles/PMC 6069403/pdf/jpmh-2018-02-e167.pdf

Gómez, Z., Landeros, P., Noa, M., Patricio, S. (2017) Consumo de alcohol, tabaco y otras drogas en jóvenes universitarios. Revista de Salud Pública y Nutrición, 16(4), 1-9. DOI: https://doi.org/10.29105/respyn16.4-1

González, P., Hernández, E., Rodríguez, L., Castillo, R., Salazar, J., y Camacho J. (2019). Percepción de riesgo ante el consumo de alcohol y tabaco en estudiantes de ciencias de la salud de Saltillo. Enfermería Global. 2, 398-410. DOI: https://doi.org/10.6018/eglobal.18.4.351381 
Herrera-Chávez, K.,Linares-Rubio, M., y DíazBarajas, D. (2018). Ambiente familiar e influencia social asociados al consumo de drogas ilegales y alcohol en adolescentes. Revista de Educación y Desarrollo.46: 61-71. Disponible en http://www.cucs.udg.mx/revistas/edu_desarrollo /anteriores/46/46_Herrera.pdf

Jiménez, L., Menéndez, S., \& Hidalgo, M. V. (2012). Un análisis de los acontecimientos vitales estresantes durante la adolescencia. Apuntes de Psicología, 30(1-3): 523-531. Disponible en https://pdfs.semanticscholar.org/18a1/30c9f1472 077466c0b605641afbcd981a7c5.pdf

Jiménez-Muro, A., Beamonte, A., Marqueta, A., Gargallo, P., Nerín, I. (2009). Consumo de drogas en estudiantes universitarios de primer curso. Revista Adicciones. 21(1), 21-28. Disponible en https://www.redalyc.org/pdf/2891/28912288200 4.pdf

Yadav, A.K., Khanuja, R.K. y Vegala, N.R. (2020). Gender differences in driving control of young alcohol-impaired drivers. Drug and Alcohol Dependence. 213. Disponible en: https://pubmed.ncbi.nlm.nih.gov/32498031/

Maturana, A. (2011). Consumo de alcohol y drogas en adolescentes. Revista Médica Clínica Las Condes, 22 (1): 98-109. DOI: 10.1016/S07168640(11)70397-2.

Disponible en:https://www.sciencedirect.com/science/article /pii/S0716864011703972

Medina-Mora, M. (2007). Mexicans and alcohol: patterns, problems and policies. Addiction. 102(7):1041-1045. DOI: $10.1111 / \mathrm{j} .1360$ 0443.2007.01857.x.

Montoya, V., Cunningham, J., Brans, B., Strike, C. y Miotto, W. (2009). Consumo percibido y uso de drogas lícitas e ilícitas en estudiantes universitarios de Medellín, Colombia. Revista Latino-Americana Enfermagem, 17, 886-892. Disponible en https://www.redalyc.org/pdf/2814/28142191302 $0 . p d f$
OMS. Organización Mundial de la Salud. (2016). La dimensión de salud pública del problema mundial de las drogas. Disponible en https://apps.who.int/gb/ebwha/pdf_files/EB140/ B140_29-sp.pdf

OMS. Organización Mundial de la Salud. (2018). Alcohol. Disponible en https://www.who.int/es/news-room/factsheets/detail/alcohol

Ortega-Ceballos, P., Terrazas-Meraz, M., Arizmendi-Jaime, E., y Tapia-Domínguez, M. (2018). Conocimientos, actitudes y factores asociados al consumo de tabaco en estudiantes universitarios de enfermería. Enfermería Universitaria, 15(2): 159-171. Disponible en http://www.scielo.org.mx/pdf/eu/v15n2/23958421-eu-15-02-159.pdf

Otten, R., Engels, R., Van de Ven, M., and Bricker, J. (2007). Parental Smoking and Adolescent Smoking Stages: The Role of Parents' Current and Former Smoking, and Family Structure. Journal of Behavioral Medicine, 30(2): 143-154. DOI: 10.1007/s10865-006-9090-3.

Ordás, B., Martínez, S., Casado, I., Bárcena, C., Álvarez, M.J., Fenandez, D. (2017) Consumo de tabaco en estudiantes universitarios de Ciencias de la Salud estudio de prevalencia. Tiempos de Enfermería y Salud. 2: 49-57. Disponible en: https://dialnet.unirioja.es/servlet/articulo?codigo $=6319011$

Plá, A., Elizarde, M., Cárdenas, E., Solares, J., \& Nieves, B. (2016). Tabaquismo: valores e integralidad. Revista Médica Electrónica, 38 (3), 460-469.

Disponible en http://www.revmedicaelectronica.sld.cu/inde x.php/rme/article/view/1526

Peña, G y Ávila, R. (2016). Pros, contras, resiliencia motivaciones de consumo de alcohol y tabaco en jóvenes de preparatoria del sur de Sinaloa. Revista Conjeturas Sociológicas. 91-112. Disponible http://ri.ues.edu.sv/id/eprint/14573/1/6.pdf

Poscia, A., Parente, P., Frisicale, E. M., Teleman, A. A., de Waure, C., Di Pietro, M. L., \& Di Pietro, 
M. L. (2015). Risky behaviours among university students in Italy. Annali dell'Istituto superiore di sanita. 51(2): 111-115. Disponible en: https://doi.org/10.4415/ANN_15_02_07

Puig-Nolasco, A., Cortaza-Ramirez, L., y Pillon, S. (2011). Consumo de alcohol entre estudiantes mexicanos de medicina. Rev. Latino-Am. Enfermagem. 19: 714-721. Disponible en: https://www.scielo.br/pdf/rlae/v19nspe/08.pdf

Reyes-Ríos. L., Camacho-Rodríguez, D., FerrelBallestas, L., Ferrel-Ortega, F y Bautista-Pérez, F. (2018). Diferencias en el consumo de tabaco en estudiantes según el sexo universitarios. Revista Cubana de Enfermería. 34(3):612-623. Disponible

en http://www.revenfermeria.sld.cu/index.php/enf/a rticle/view/1479/376.

Rodríguez, L., Alonso, B., Alonso, M., Alonso, M., Armendáriz, N., y Oliva, N. (2016). Consumo de alcohol y tabaco en adolescentes. SMAD. Revista Electrónica en Salud Mental, Alcohol y Drogas. 12(4): 200-206. https://doi.org/10.11606/issn.18066976.v12i4p200-206.

Ruiz-Risueño, A., Ruiz-Juan, F., y Zamarripa, I. (2012). Alcohol y tabaco en adolescentes españoles y mexicanos y su relación con la actividad físico-deportiva y la familia. Rev Panam Salud Publica. 31(3): 211-20. Disponible en:

https://www.scielosp.org/pdf/rpsp/2012.v31n3/2 $11-220 / \mathrm{es}$

Salcedo, A., Palacios, X., y Espinosa, F. (2011). Consumo de alcohol en jóvenes universitarios. Avances en Psicología Latinoamericana, 29(1): 77 - $97 . \quad$ Disponible en http://revistas.urosario.edu.co/index.php/apl/arti cle/view/640

Sánchez-Hoil, A., Andueza-Pech, M., SantanaCarvajal, A, Hoil-Santos, J, y CuFarfán-López, J. (2017). Revista Biomédica. 28(1): 11-27. Disponible en https://doi.org/10.32776/revbiomed.v28i1.552
Simón, M. J., Fuentes R. M., Garrido, M., Serrano P., Díaz, M. J. y Yubero, S. (2019). Perfil de consumo de drogas en adolescentes. Factores protectores. Medicina de Familia, 46(1): 33-40. Doi: 10.1016/j.semerg.2019.06.001. Disponible en: file:///C:/Users/hizoy/Downloads/Simon_perfilc onsumoyf.protectoresSEMERGEN.pdf

Strunin, L., Díaz-Martínez, A., Díaz-Martínez, R., Heeren, T., Chen, C., Winter, M., Kuranz, S., Hernández-Ávila, C., Fernández-Varela, H., and Solís-Torres, C. (2017). Age of onset, current use of alcohol, tobacco or marijuana and current polysubstance use among male and female Mexican students. Alcohol and Alcoholism. 52(5): 564-571. Doi: 10.1093/alcalc/agx027

Telumbre, J y Sánchez, B. (2015). Percepción de Barreras y Beneficios del Consumo de Alcohol en Adolescentes. Nure Investigación. 12(7): 1$10 . \quad$ Disponible en https://www.nureinvestigacion.es/OJS/index.php /nure/article/view/99/87

Telumbre-Terrero, J.Y., Esparza-Almanza, S.E., Alonso-Castillo, B.A y Alonso-Castillo, M.T. (2016). Consumo de alcohol y tabaco en estudiantes de enfermería. Rev. Enfermería Actual en Costa Rica, 30, 1-16. DOI: http://dx.doi.org/10.15517/revenf.v0i30.22020

Tiburcio, M., Rosete-Mohedano, M., Natera, G., Martínez, N., Carreño, S. Pérez, D. (2016). Validez y confiabilidad de la prueba de detección de consumo de alcohol, tabaco y sustancias (ASSIST) en estudiantes universitarios. Adicciones. 28 (1): 19-27. Disponible en http://www.adicciones.es/index.php/adicciones/a rticle/view/786/750

Tirado, A., Alvarez, M., Velásquez, J., Gómez, L., Ramírez, C., Vargas, A. (2012). Prevalencia y factores de riesgo para el consumo y dependencia de drogas en estudiantes de una universidad de Medellín, Colombia, 2009. Rev. Fac. Nac. Salud Pública. 30 (1): 38-44. Disponible en: file:///C:/Users/hizoy/Downloads/DialnetPrevalenciaYFactoresDeRiesgoParaElConsumo YDependen-5079636.pdf 
Valdez-Rodríguez, B., Rodríguez-Olivas, M., Hernández-Bernadett, J., y Terrazas-Altamirano, D. (2019). Características de fumadores universitarios. ConCiencia Tecnológica. 58: 14$20 . \quad$ Disponible en https://dialnet.unirioja.es/ejemplar/537666

Villarreal, M., Sánchez, J., Musitu, G. (2013). Análisis psicosocial del consumo de alcohol en adolescentes mexicanos. Universitas Psychologica. 12 (3): 857 873. Disponible en: https://www.redalyc.org/pdf/647/64730275017.

pdf

Villatoro,J., Medina-Mora. M., Martín del Campo, R., Fregoso, D., Bustos, N., Resendiz. E., Mujica, R., Bretón, M., Soto, S., y Cañas, V. (2016). El consumo de drogas en estudiantes de México: tendencias y magnitud del problema. Salud Mental. 39(4): 193-203. Disponible en: https://www.medigraphic.com/cgibin/new/resumen.cgi?IDARTICULO=67799

Zamora-Mendoza, A., Hernández-Castañón, M., Álvarez-Aguirre, A., Garza-González, B., y Gallegos-Torres, R. (2013). Prevalencia de sustancias adictivas y estilos de vida en estudiantes universitarios. Revista Ciencia@UAQ. Disponible en https://www.uaq.mx/investigacion/revista_cienci a@uaq/ArchivosPDF/v6-n2/15Articulo.pdf 